\title{
Elastocaloric effect with a broad temperature window and low energy loss in a nanograin Ti-44Ni-5Cu-1Al (at.\%) shape memory alloy
}

\author{
Hong Chen, ${ }^{1,4}$ Fei Xiao, ${ }^{1,3, *}$ Zhu Li, ${ }^{1}$ Xuejun Jin $\odot,{ }^{2, \dagger}$ Lluís Mañosa, ${ }^{4}$ and Antoni Planes ${ }^{4}$ \\ ${ }^{1}$ State Key Laboratory of Metal Matrix Composite, School of Materials Science and Engineering, Shanghai Jiao Tong University, \\ 800 Dong Chuan Road, Shanghai 200240, People's Republic of China \\ ${ }^{2}$ Institute of Advanced Steels and Materials, School of Materials Science and Engineering, Shanghai Jiao Tong University, \\ Shanghai 200240, People's Republic of China \\ ${ }^{3}$ Department of Materials Science and Engineering, Graduate School of Engineering, Osaka University, 2-1, \\ Yamada-oka, Suita, Osaka 565-0871, Japan \\ ${ }^{4}$ Departament de Física de la Matèria Condensada, Facultat de Física, Universitat de Barcelona, Martí i Franquès 1, \\ E-08028 Barcelona, Catalonia, Spain
}

(Received 29 October 2020; revised 24 December 2020; accepted 4 January 2021; published 26 January 2021)

\begin{abstract}
Superelastic Ti-Ni-based shape memory alloys are promising elastocaloric materials for solid-state refrigeration. In this paper, we studied the mechanical properties and elastocaloric effect of a severely cold rolled Ti-44Ni-5Cu-1Al (at.\%) alloy composed of nanograins $(\sim 5-16 \mathrm{~nm})$. The alloy exhibits partial stress-induced martensitic transformation with a large quasilinear reversible strain of $\sim 3.4 \%$ and a small energy dissipation of $\sim 1.6 \mathrm{MJ} / \mathrm{m}^{3}$ when a maximum stress of $1 \mathrm{GPa}$ is applied. The temperature decrease induced by adiabatic removal of a stress of $1 \mathrm{GPa}$ is of about $-5 \mathrm{~K}$, which is mainly due to the reverse transition occurring during unloading. The effective working temperature window of elastocaloric effect larger than $200 \mathrm{~K}$. The coefficient of performance of this alloy reaches $\sim 13$, which is higher than values reported so far for Ti-Ni alloys.
\end{abstract}

DOI: 10.1103/PhysRevMaterials.5.015201

\section{INTRODUCTION}

New cooling devices using giant caloric effects in solids have attracted much attention in recent years [1,2]. They are environmentally friendly and are expected to replace the most commonly used vapor-compression refrigeration technology that uses environmentally harmful fluids $[3,4]$. The elastocaloric effect (eCE) in near equiatomic Ti-Ni shape memory alloys (SMAs) is considered to be among the most promising caloric effect [5,6] since it can be induced by applying moderate uniaxial stresses that are feasible in refrigeration devices. The eCE in Ti-Ni SMAs originate from the latent heat associated with the stress-induced martensitic transformation (MT) that causes significant entropy change $\left(\Delta S_{\text {iso }}\right)$ and temperature change $\left(\Delta T_{\text {adi }}\right)$ under isothermal and adiabatic application/removal of stress, respectively [7].

A wide working temperature window (WTW) is a desired property of cooling devices and, therefore, refrigerant materials need to accommodate a wide temperature range in practical applications. The effective WTW of conventional Ti-Ni SMAs is less than $30 \mathrm{~K}$ [8], which is clearly insufficient for many applications. This is a consequence of the fact that the first-order MT in Ti-Ni SMAs is very sharp.

Many studies have been conducted aimed to broaden the WTW of eCE in Ti-Ni SMAs. The addition of third elements has little effect on the WTW, although it affects the transformation temperature [9]. Ahadi and Sun found that in Ti-50.9Ni (at.\%) SMAs the temperature ranges broaden dras-

\footnotetext{
*Corresponding author: xfei@sjtu.edu.cn

†Corresponding author: jin@sjtu.edu.cn
}

tically as the grain size decreases [10]. Such a phenomenon was also reported in a cold-drawn Ti-Ni microwire with the grain size of $\sim 30 \mathrm{~nm}$, whose WTW was extended to $\sim 70 \mathrm{~K}$ [11]. Therefore, we may expect that a much broader WTW could be achieved in Ti-Ni-based SMA if the grains are sufficiently small. In addition, it is expected that grain size reduction can improve the fatigue life of Ti-Ni-based SMA and decrease the hysteresis through a stress cycling $[12,13]$, which is also very important for improving eCE performances.

In the present paper, we selected the $\mathrm{Ti}-44 \mathrm{Ni}-5 \mathrm{Cu}-1 \mathrm{Al}$ (at.\%) alloy as a candidate alloy because it is known that the Ti-44Ni-5Cu-1Al with $\sim 40$-nm grain size exhibits smaller stress hysteresis and better fatigue life than Ti-Ni alloys with similar grain sizes [14]. To achieve broader WTW and substantially smaller hysteresis, heavier cold rolling (CR) was employed to obtain smaller grain sizes. The microstructure, MT, superelasticity, and eCE were systematically studied in the prepared severely cold-rolled Ti-44Ni-5Cu-1 Al(at.\%).

\section{EXPERIMENTAL DETAILS}

A polycrystalline Ti-44Ni-5Cu-1Al (at.\%) ingot was fabricated by vacuum induction melting. An $\sim 4$-mm thickness slab was cut from the ingot and was hot rolled at $1123 \mathrm{~K}$ to $\sim 70 \%$ thickness reduction and further severely $\mathrm{CR}$ to $\sim 60 \%$ thickness reduction. We refer to the specimens in this state as nanograin $\mathrm{Ti}-44 \mathrm{Ni}-5 \mathrm{Cu}-1 \mathrm{Al}$ (at.\%) alloy (NGTNCA). Compared with the previously studied CR specimens [14], the present NG-TNCA underwent heavier rolling. Therefore, smaller grain size and higher density of defects were expected to be formed in the NG-TNCA. 


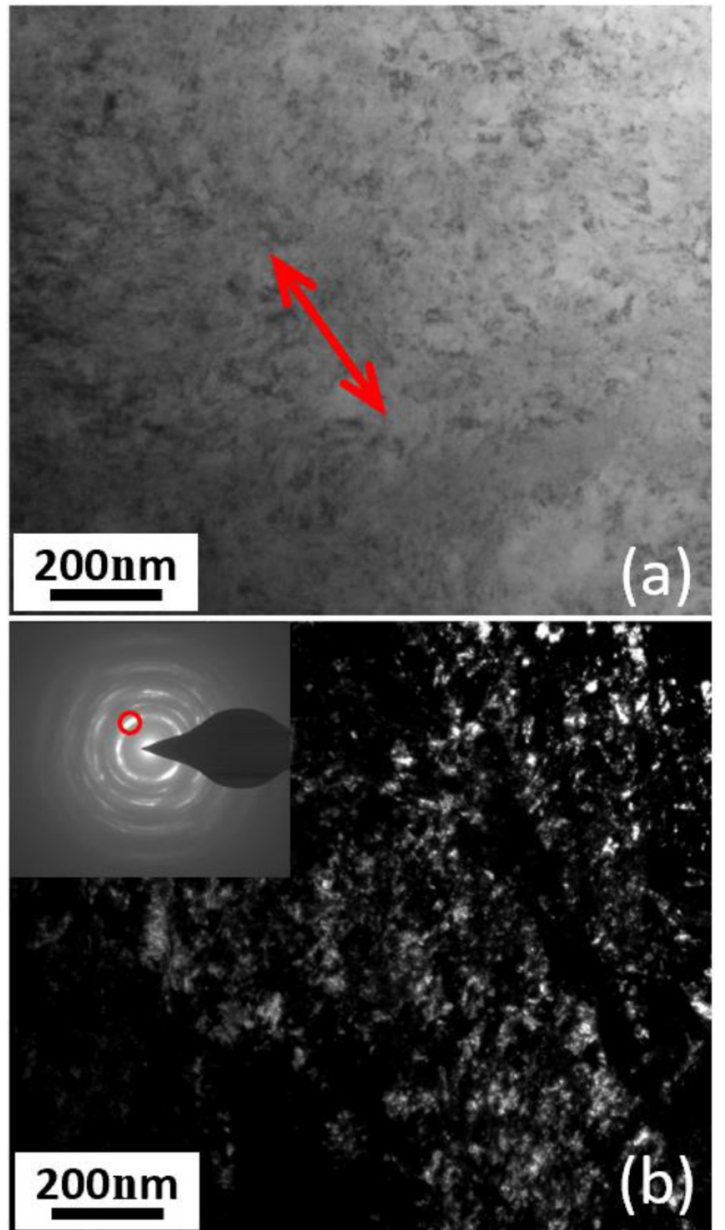

FIG. 1. (a) Bright-field image, (b) dark-field image and corresponding diffraction pattern for the nanograin $\mathrm{Ti}-44 \mathrm{Ni}-5 \mathrm{Cu}-1 \mathrm{Al}$ (at.\%) alloy. The arrow indicates the rolling direction, and the circle indicates the position of the objective aperture with which the darkfield image is taken.

The alloy microstructure was observed at room temperature $(\sim 293 \mathrm{~K})$ using a JEOL $2100 \mathrm{~F}$ transmission electron microscope. Transformation characteristics were determined by differential scanning calorimetry (DSC) with a cooling/heating rate of $10 \mathrm{~K} / \mathrm{min}$ and by standard four-point contact electrical resistivity (ER) at a cooling/heating rate of $2 \mathrm{~K} / \mathrm{min}$. In situ structural evolution under tensile stress was evaluated by a Rigaku RAPID II transmission x-ray diffraction (XRD) with a Mo target. Volume specific heat $\left(C_{E}\right)$ was measured by a relaxation method using a Quantum Design physical property measurement system (PPMS). The dynamic Young's modulus was obtained using a TA instrument Q800 dynamic mechanical analyzer in the tensile mode.

For mechanical tests and elastocaloric temperature change measurements, dog-bone shaped specimens cut along the rolling direction were used. These tests were performed by using an INSTRON-5969 mechanical testing machine equipped with a temperature control system. The strain was determined by a video extensometer, and the temperature change was monitored by a J-type thermocouple welded onto the surface of the specimen.
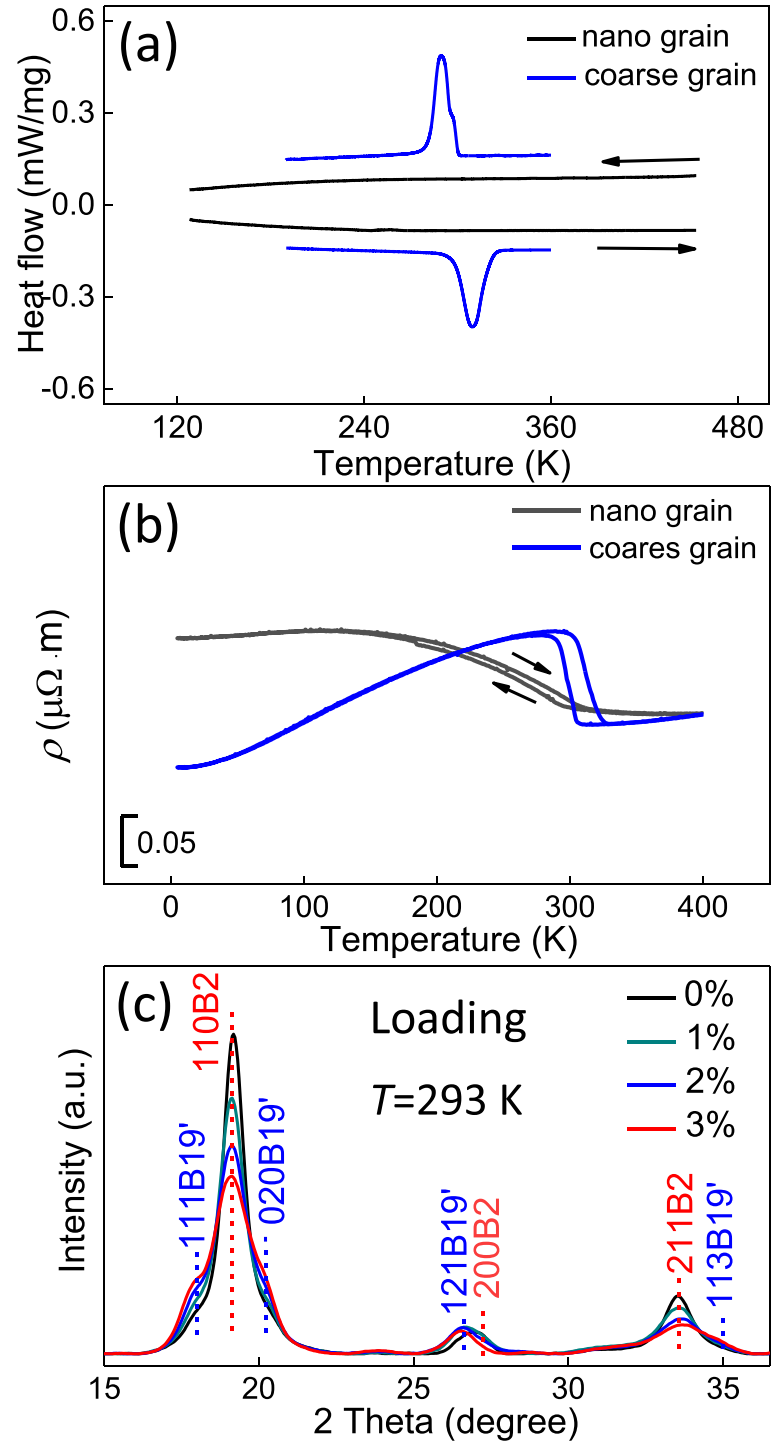

FIG. 2. (a) DSC cooling and heating curves measured at a rate of $10 \mathrm{~K} / \mathrm{min}$, (b) temperature dependence of the electrical resistivity $(\rho)$ for the nanograins (black line) and coarse grain (blue line) Ti$44 \mathrm{Ni}-5 \mathrm{Cu}-1 \mathrm{Al}$ (at.\%) alloys, (c) in situ $\mathrm{x}$-ray diffraction profiles at different tensile strains during the loading process for the nanograin Ti-44Ni-5Cu-1Al (at.\%) alloy.

\section{RESULTS AND DISCUSSION}

Figure 1 shows a bright-field image and the corresponding dark-field image taken from the marked reflections of the corresponding diffraction pattern. A typical elongated microstructure along the rolling direction (indicated by a red arrow) was observed in the images $[15,16]$. The grain size is estimated from the dark-field image to be in the range of 5-16 nm. This microstructure is the reason why we call the present specimen as NG-TNCA, which exhibits much smaller grain size than that of previously studied CR specimens [14].

The thermal-induced MT in the NG-TNCA was checked by DSC [Fig. 2(a)] and ER [Fig. 2(a)]. Compared with the coarse-grains sample, no significant transition peak appeared both in the DSC heat flow curves during cooling and heating 

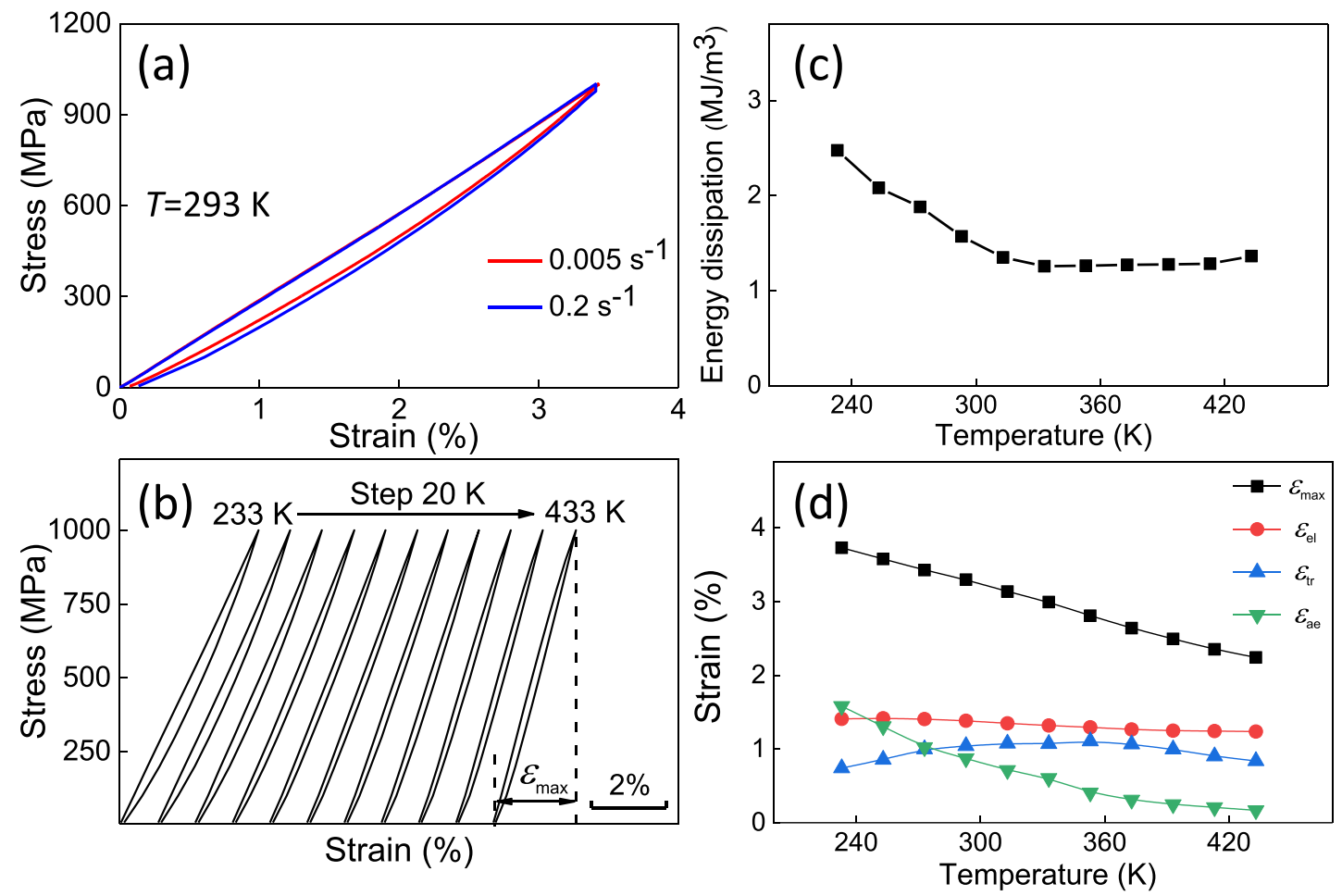

FIG. 3. (a) Stress-strain curves with strain rate of $0.005 \mathrm{~s}^{-1}$ (red line) or $0.2 \mathrm{~s}^{-1}$ (blue line), (b) stress-strain curves at different testing temperatures with a $0.005-\mathrm{s}^{-1}$ strain rate the maximum strain $\left(\varepsilon_{\max }\right)$ is defined in the figure, (c) temperature dependences of the mechanical energy dissipation calculated by integrating the areas within the loops in (b), and (d) temperature dependences of the maximum strain $\left(\varepsilon_{\max }\right)$, transformation strain $\left(\varepsilon_{\mathrm{tr}}\right)$, anelastic strain $\left(\varepsilon_{\mathrm{ae}}\right)$, and elastic strain $\left(\varepsilon_{\mathrm{el}}\right)$ for the nanograin Ti-44Ni-5Cu-1Al (at.\%) alloy.

processes in the NG-TNCA, implying that significant firstorder MT was suppressed. This is in contrast with the broad peak detected in the previously studied CR specimen [14]. This means that the MT was suppressed to a large extent by heavier rolling. Although the MT in the NG-TNCA was not observed by DSC, it was detected by ER, which suggest that the MT occurs partially. The MT of the NG-TNCA spreads over in a wide temperature range of $150-323 \mathrm{~K}$ with a narrow temperature hysteresis of $\sim 10-\mathrm{K}$ [black curves in Fig. 2(b)]. This transformation behavior is significantly different from that found in the coarse-grained $(\sim 10 \mu \mathrm{m})$ specimen [blue curves in Fig. 2(b)] with a narrower transformation range of $295-325 \mathrm{~K}$ and a wider temperature hysteresis of $\sim 17 \mathrm{~K}$ [14].

The stress-induced MT in the NG-TNCA was detected by in situ XRD measurements. Figure 2(c) shows the XRD profiles during the loading process. As the tensile strain increases, the intensities of the $110_{\mathrm{B} 2}, 211_{\mathrm{B} 2}$, and $220_{\mathrm{B} 2}$ peaks slightly decrease, and the intensities of the $111_{\mathrm{B} 19^{\prime}}, 020_{\mathrm{B} 19^{\prime}}, 121_{\mathrm{B} 19^{\prime}}$, and $113_{\mathrm{B} 19^{\prime}}$ peaks slightly increase. This result confirms that a weak stress-induced B2-B19' MT occurs in the NG-TNCA. The reverse transformation in the stress-removing process was also confirmed (not shown here). Therefore, it is expected that the NG-TNCA display noticeable eCE.

Figure 3(a) shows stress-strain curves of the NG-TNCA obtained at a strain rate of $0.005 \mathrm{~s}^{-1}$ (red curve) at $293 \mathrm{~K}$. The NG-TNCA shows quasilinear superelasticity with $3.4 \%$ strain under $1 \mathrm{GPa}$, and the residual strain is negligibly small $(\sim 0.06 \%)$. In contrast to the typical superelastic behavior of Ti-Ni SMAs, no superelastic plateau is observed, and the hys- teresis area is extremely small [17]. In a previous work [14], a noticeable irreversible strain $(\sim 0.25 \%)$ was reported in a CR specimen induced by a loading stress of $1 \mathrm{GPa}$, indicating that the heavier rolling process applied to the present specimen significantly improves the mechanical properties.

Figure 3(b) shows stress-strain curves obtained at a strain rate of $0.005 \mathrm{~s}^{-1}$ at different test temperatures. The curves are shifted horizontally for the sake of clarity. The NG-TNCA shows quasilinear superelasticity over a broad temperature range of $\sim 200 \mathrm{~K}$. The strain under $1 \mathrm{GPa}$ is between $2.6 \%$ and $3.4 \%$, depending on test temperature. Residual strain is negligibly small at any temperature examined even below the $A_{\mathrm{f}}$ temperature of $323 \mathrm{~K}$. It is noted that the shape of the hysteresis loops is hardly affected by temperature. The energy dissipation $\left(\Delta E_{\text {diss }}\right)$ caused by stress hysteresis can be obtained from integration of the area of stress-strain loops. The temperature dependences of $\Delta E_{\text {diss }}$ are shown in Fig. 3(c). $\Delta E_{\text {diss }}$ gradually decreases on cooling down to $M_{\mathrm{s}}$ [ $\sim 313 \mathrm{~K}$ obtained from Fig. 2(b)] and then increases substantially. These results suggest that the total strain $\left(\varepsilon_{\max }\right)$ under $1 \mathrm{GPa}$ consists of three contributions: elastic strain caused by the elastic deformation $\left(\varepsilon_{\mathrm{el}}\right)$, transformation strain originated from the stress-induced MT $\left(\varepsilon_{\text {tr }}\right)$, and anelastic strain arising from the rearrangement of martensite variants $\left(\varepsilon_{\mathrm{ae}}\right) \cdot \varepsilon_{\mathrm{el}}$ can be calculated by using the macroscopic Young's modulus (the data are shown in the Supplemental Material [18]). We assumed that the elastocaloric temperature change $\Delta T_{\text {adi }}^{\text {exp }}$ measured by the thermocouple [shown in Fig. 4(b)] is proportional to the amount of $\varepsilon_{\mathrm{tr}}$. In the case of 


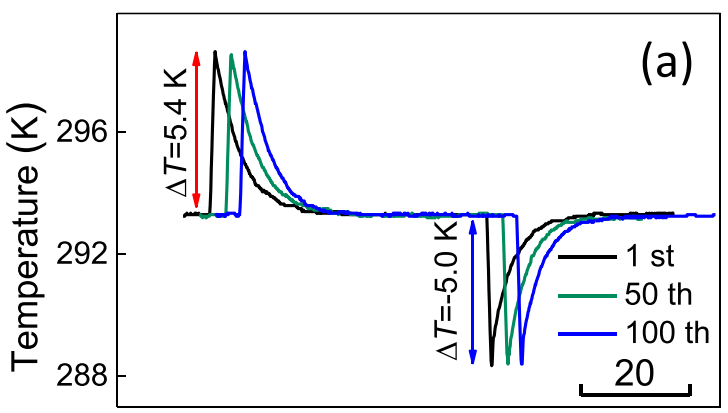

Time (s)

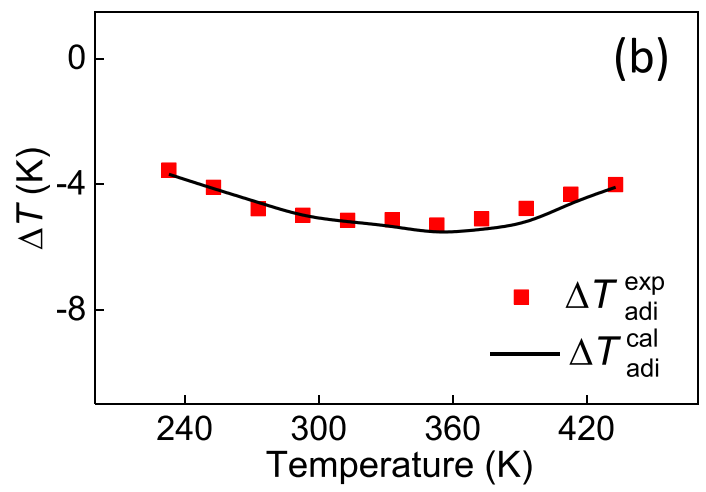

FIG. 4. (a) Time dependence of the temperature of the specimen measured in the loading, holding, unloading, and holding processes with a fast loading/unloading rate of $0.2 \mathrm{~s}^{-1}$, (b) testing temperature dependence of the experimental adiabatic temperature change $\Delta \mathrm{T}_{\text {adi }}^{\exp }$ during the stress fast removing process $\left(0.2 \mathrm{~s}^{-1}\right)$ from $1 \mathrm{GPa}$. The solid line is the calculated adiabatic temperature change $\Delta \mathrm{T}_{\text {adi }}^{\text {cal }}$ calculated from isothermal stress-strain curves using the Maxwell relation for the nanograin $\mathrm{Ti}-44 \mathrm{Ni}-5 \mathrm{Cu}-1 \mathrm{Al}($ at.\%) alloy.

Ti-44Ni-5Cu-1 Al (at.\%) sheets with a larger grain size [19], the temperature change is $-25.4 \mathrm{~K}$ when $\varepsilon_{\text {tr }}$ of $5.3 \%$. Therefore, $\varepsilon_{\text {tr }}$ for NG-TNCA is estimated to be $\Delta T_{\text {adi }}^{\exp } \times$ $5.3 \% / 25.4 \mathrm{~K}$. Then the remaining contribution to $\varepsilon_{\max }$ should be $\varepsilon_{\mathrm{ae}}$. The calculated results are summarized in Fig. 3(d). $\varepsilon_{\text {el }}$ (red cycle) slightly increases with decreasing temperature. This is probably due to the softening of the parent phase's elastic constants and the formation of martensite on cooling [20]. $\varepsilon_{\text {tr }}$ (blue triangle) shows a maximum near the $A_{\mathrm{f}}$ temperature [ $\sim 323 \mathrm{~K}$ obtained from Fig. 2(b)]. This is a reasonable behavior since the stress requited to induce the MT increases as temperature increases above $A_{\mathrm{f}}$, and the fraction of the parent phase, which can transform to the martensite, decreases as temperature decreases below $A_{\mathrm{f}}$ due to thermally induced MT. $\varepsilon_{\mathrm{ae}}$ is small above the $A_{\mathrm{f}}$ temperature due to the existence of the residual martensite phase, which forms during the cold-rolling process. The existence of this residual martensite was confirmed by the XRD results in Fig. 2(c). The fraction of martensite increases at low temperature due to the MT and, therefore, $\varepsilon_{\mathrm{ae}}$ increases with decreasing temperature. In contrast with large grain Ti-Ni SMAs, the rearrangement of martensite variants in the NG-TNCA is reversible even at low temperature, which is probably due to the nanometer microstructure and strong texture formed by heavy cold rolling.
The elastocaloric temperature change in the NG-TNCA was studied from measurement at the high strain rate of $0.2 \mathrm{~s}^{-1}$ that approximates ensured adiabatic conditions. Stress-strain behavior is shown in Fig. 3(a) (blue curve). After loading to the maximum stress, the strain was held for $50 \mathrm{~s}$ to enable heat transfer and to achieve thermal equilibrium with the ambient temperature. Then the stress was removed. The obtained stress-strain curve at the strain rate of $0.2 \mathrm{~s}^{-1}$ is essentially similar to that under the strain rate of $0.005 \mathrm{~s}^{-1}$, although the former has a slightly larger hysteresis and the residual strain. The temperature of the specimen monitored by a thermocouple is shown in Fig. 4(a) as the black line. The adiabatic temperature change $\left(\Delta T_{\text {adi }}^{\exp }\right)$ in the loading process is $5.4 \mathrm{~K}$, and that in the unloading process is $-5.0 \mathrm{~K}$. The same test was repeated 100 times, and the temperature changes for the 50th and 100th cycles are also shown in Fig. 4(a) by green and blue curves. The temperature change in the 100th cycle is essentially the same as that of the 50th and first cycles. This is a clear corroboration of the fact that the NG-TNCA has good fatigue properties.

The temperature dependence of $\Delta T_{\text {adi }}^{\text {exp }}$ in the unloading process in the temperature range of $233-433 \mathrm{~K}$ is shown as red solid squares in Fig. 4(b). The maximum value of $\left|\Delta T_{\text {adi }}^{\exp }\right|=$ $5.3 \mathrm{~K}$ is obtained when the test temperature is $333 \mathrm{~K}$ (approximately $10 \mathrm{~K}$ above $A_{\mathrm{f}}$ ). The theoretical temperature change

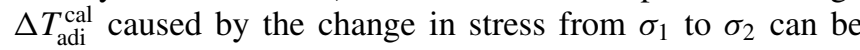
evaluated from the Maxwell relation as

$$
\Delta T_{\text {adi }}^{\text {cal }}=-\int_{\sigma_{1}}^{\sigma_{2}} \frac{T}{C_{E}}\left(\frac{\partial \varepsilon}{\partial T}\right)_{\sigma} d \sigma
$$

where $T$ is the temperature and $C_{E}$ is the volume heat capacity under stress. Using the unloading branches of stress-strain curves in Fig. 3(b), we computed $\Delta T_{\text {adi }}^{\mathrm{cal}}$ as a function of temperature, and the obtained results are shown as the solid line in Fig. 4(b). We have assumed $C_{E}$ to be independent of stress and the values measured by Quantum Design PPMS (the data are shown in the Supplemental Material [18]). The agreement between the experimental results and the calculated results is satisfactory. Such an agreement indicates that experiments performed at a $0.2-\mathrm{s}^{-1}$ strain rate are suitable to achieve adiabatic conditions and that Eq. (1) is reliable for the evaluation of the elastocaloric effect of the NG-TNCA.

Despite the fact that measured values of $\Delta T$ in the present NG-TNCA are lower than those reported for larger grain $\mathrm{Ti}-\mathrm{Ni}$, the present NG-TNCA possesses a number of advantages as a refrigerant material. The first is the wide working temperature window. The effective WTW of the eCE, which is defined as the full width at half maximum of the peak giving $\Delta T_{\text {adi }}^{\exp }$ as a function of temperature in Fig. 4(b) [21], is wider than $200 \mathrm{~K}$. This value is much larger than that of the coarse-grained Ti-Ni alloy $(\sim 12-17 \mathrm{~K})$ [7], Ti-44Ni-5Cu-1Al sheets with a $\sim 40$-nm grain size $(\sim 55 \mathrm{~K})$ [19] and cold-drawn Ti-Ni microwires with a $\sim 30$-nm grain size $(\sim 70 \mathrm{~K})$ [11]. It is even larger than the largest value reported so far for $\mathrm{Cu}-\mathrm{Zn}-\mathrm{Al}$ SMAs $(\sim 130 \mathrm{~K})[22]$.

The reason for the wide WTW is a consequence of the diffuse character of the MT that occurs within a broad temperature range as confirmed by ER in Fig. 1(b). Compared with coarse-grained samples, the MT in the nanograin sample occurs in a broader transformation temperature interval 
$\left(M_{\mathrm{s}}-M_{\mathrm{f}}\right)$. The effect of grain size on the transformation temperatures $M_{\mathrm{s}}$ and $M_{\mathrm{f}}$ can be understood as follows. In the case of thermoelastic MT, the total free-energy change between parent and martensitic phases can be estimated as

$$
\Delta G=\Delta G_{\mathrm{ch}}+\Delta E_{\mathrm{el}}+\Delta E_{\mathrm{irr}},
$$

where $\Delta G_{\mathrm{ch}}$ is the chemical free-energy change provided by the the Gibbs free-energy difference between parent and martensite phases, $\Delta E_{\mathrm{el}}$ is the elastic strain energy associated with the transition-induced elastic strain, and $\Delta E_{\text {irr }}$ is the irreversible energy associated with the interface propagation and defect generation. Although $\Delta G_{\mathrm{ch}}$ provides the driving force for the MT, $\Delta E_{\mathrm{el}}$ and $\Delta E_{\mathrm{irr}}$ represent the energy barrier that opposes transformation. Thus, undercooling or stress increase is necessary for the transition to proceed. The higher-energy barrier in nanograin samples is the reason for a lower transformation temperature of the NG-TNCA than in the coarse-grained sample. Actually, the MT consists of two stages: nucleation and growth. There are many grain boundaries and defects in NG-TNCA that provide nucleation sites and, thus, the $M_{\mathrm{s}}$ of the NG-TNCA is only $12 \mathrm{~K}$ lower than in the corresponding coarse-grained sample. However, the martensite growth in each nanograin is severely prevented by grain boundaries and defects, which leads to a large decrease in $M_{\mathrm{f}}$. Thus, a broad transformation interval $\left(M_{\mathrm{s}}-M_{\mathrm{f}}\right)$ is obtained in the NG-TNCA. In addition, after cold rolling, the microstructure of the NG-TNCA is highly inhomogeneous and, consequently, the MT temperature in the different parts of the sample can be very different, which also contributes to the broadening of the transformation interval.

The ultrafine structure prevents plastic deformation to occur even at high temperatures and the reversible rearrangement of martensite variants even at low temperatures, and these are also contributed to the wide WTW.

The second advantage for the NG-TNCA is the small stress hysteresis. $\Delta E_{\text {diss }}$ caused by stress hysteresis is $1.6 \mathrm{MJ} / \mathrm{m}^{3}$ at $293 \mathrm{~K}$. The corresponding irreversible temperature change $\Delta T_{\text {irr }}$ associated with this dissipation can be estimated as $\Delta T_{\text {irr }}=\frac{1}{C_{E}} \Delta E_{\text {diss }}[23,24]$ to be $0.4 \mathrm{~K}$, which agrees with the difference in the absolute value of $\Delta T_{\text {adi }}^{\exp }$ measured in the loading and unloading processes $(0.4 \mathrm{~K}=5.4-5.0 \mathrm{~K})$. Although the $\Delta E_{\text {diss }}$ increases to $2.5 \mathrm{MJ} / \mathrm{m}^{3}$ as temperature decreases to $233 \mathrm{~K}$, it is much smaller than that in conventional Ti-Ni SMAs $\left(\sim 22 \mathrm{MJ} / \mathrm{m}^{3}\right)$ [25]. The small $\Delta E_{\text {diss }}$ in NG-TNCA is an important factor achieved as the stable eCE. In fact, there are three main reasons for the lower hysteresis loss in the present system: The first is the smaller volume frac- tion of the MT compared with specimens with larger grains; the second is the improvement of the alloy strength induced by severely cold rolling; and the third is the improvement of lattice compatibility by $\mathrm{Cu}$ addition that leads to considerable reduction of plastic deformation $[19,26]$.

The extremely small hysteresis indicated that the NGTNCA should have high COP. COP is defined as the ratio of the useful heat removed from the cold reservoir $(Q)$ to the input work $(W)$. That is, $\mathrm{COP}=|Q / W|[27,28]$. The value of $Q$ can be estimated as $Q \cong C_{p} \Delta T_{\text {adi }}$, and the value of $W$ can be obtained from integration of stress-strain curves. The maximum value of COP is obtained to be $\sim 13$ at $333 \mathrm{~K}$. This value is higher than the COP for the conventional Ti-Ni alloys reported in previous papers $(\sim 5.3)[28]$ and is higher than the COP of the Ti-44Ni-5Cu-1Al (at.\%) alloy with a 40-nm grain size ( 9.6) [19]. After severely cold rolling, the NG-TNCA shows partial stress-induced MT. Therefore, a moderate strain value and $\Delta T_{\text {adi }}$ have been obtained during the tensile process. This means that the $Q$ value of NG-TNCA is small. However, the extremely small hysteresis of NG-TNCA causes a drastic decreasing of $W$ and, consequently, the NG-TNCA has the highest value of COP compared with other conventional coarse-grained Ti-Ni alloys.

\section{SUMMARY AND CONCLUSIONS}

To summarize, in this paper, we have performed a comprehensive study on the MT, superelasticity, and eCE of a nanograin Ti-44Ni-5Cu-1 Al (at.\%) alloy. Quasilinear superelasticity with recoverable strains of $3.4 \%$ and extremely small $\Delta E_{\text {diss }}$ of $\sim 1.6 \mathrm{MJ} / \mathrm{m}^{3}$ was obtained under a tensile stress up to $\sim 1 \mathrm{GPa}$. A stable and highly effective (COP $\sim 13$ ) eCE characterized by an adiabatic temperature decrease reaching $\sim-5 \mathrm{~K}$ and an effective WTW of more than $200 \mathrm{~K}$ has been obtained.

\section{ACKNOWLEDGMENTS}

This work was funded by the National Natural Science Foundation of China (Grants No. 52022055, No. 52031005, and No. 51871151). This research was also supported by Natural Science Foundation of Shanghai (Grant No. 20ZR1428800), Osaka University's International Joint Research Promotion Program, and SJTU-OU Strategic Partnership Development Fund. A part of financial support come from the Program of China Scholarships Council (Grant No. 201906230103). L.M. and A.P. acknowledge support from the Spanish Ministry of Science, Project No. MAT2016-75823-R.
[1] J. Tušek, K. Engelbrecht, D. Eriksen, S. Dall'Olio, J. Tušek, and N. Pryds, Nat. Energ. 1, 16134 (2016).

[2] S. X. Qian, Y. L. Geng, Y. Wang, J. Z. Ling, Y. H. Hwang, R. Radermacher, I. Takeuchi, and J. Cui, Int. J. Refrig. 64, 1 (2016).

[3] J. Tušek, K. Engelbrecht, R. Millán-Solsona, L. Mañosa, E. Vives, L. P. Mikkelsen, and N. Pryds, Adv. Energ. Mater. 5, 1500361 (2015).

[4] J. M. Calm, Int. J. Refrig. 31, 1123 (2008).

[5] L. Mañosa and A. Planes, Adv. Mater. 29, 1603607 (2017).
[6] J. Cui, Y. M. Wu, J. Muehlbauer, Y. H. Hwang, R. Radermacher, S. Fackler, M. Wuttig, and I. Takeuchi, Appl. Phys. Lett. 101, 073904 (2012).

[7] D. Soto-Parra, E. Vives, L. Mañosa, J. A. Matutes-Aquino, H. Flores-Zúñiga, and A. Planes, Appl. Phys. Lett. 108, 071902 (2016).

[8] S. Miyazaki, K. Otsuka, and Y. Suzuki, Scr. Metall. 15, 287 (1981).

[9] J. Frenzel, A. Wieczorek, I. Opahle, B. Maaß, R. Drautz, and G. Eggeler, Acta Mater. 90, 213 (2015). 
[10] A. Ahadi and Q. P. Sun, Appl. Phys. Lett. 103, 021902 (2013).

[11] X. X. Zhang, M. F. Qian, X. J. Zhu, C. Shang, and L. Geng. APL Mater. 6, 036102 (2018).

[12] H. Chen, F. Xiao, X. Liang, Z. Li, Z. Li, X. Jin, N. Min, and T. Fukuda, Scr. Mater. 162, 230 (2019).

[13] H. L. Hou, E. Simsek, T. Ma, N. S. Johnson, S. X. Qian, C. Cissé, D. Stasak, N. A. Hasan, L. Zhou, Y. H. Hwang, R. Radermacher, V. I. Levitas, M. J. Kramer, M. A. Zaeem, A. P. Stebner, R. T. Ott, J. Cui, and I. Takeuchi, Science 366, 1116 (2019).

[14] H. Chen, F. Xiao, X. Liang, Z. Li, X. Jin, and T. Fukuda, Acta Mater. 158, 330 (2018).

[15] A. Ahadi, Y. Matsushita, T. Sawaguchi, Q. P. Sun, and K. Tsuchiya, Acta Mater. 124, 79 (2017).

[16] S. D. Prokoshkin, I. Y. Khmelevskaya, S. V. Dobatkin, I. B. Trubitsyna, E. V. Tatyanin, V. V. Stolyarov, and E. A. Prokofiev, Acta Mater. 53, 2703 (2005).

[17] X. Zhang, P. Feng, Y. He, T. Yu, and Q. Sun, Int. J. Mech. Sci. 52, 1660 (2010).

[18] See Supplemental Material at http://link.aps.org/supplemental/ 10.1103/PhysRevMaterials.5.015201 for temperature dependence of Young's modulus $E$ and temperature dependence of the volume heat capacity $C E$ of the nanograin $\mathrm{Ti}-44 \mathrm{Ni}-5 \mathrm{Cu}-1 \mathrm{Al}$ alloy.

[19] H. Chen, F. Xiao, X. Liang, Z. Li, Z. Li, X. Jin, and T. Fukuda, Acta Mater. 177, 169 (2019).

[20] Y. Liu and H. Xing, J. Alloys Compd. 270, 154 (1998).

[21] H. Hua, J. M. Wang, C. B. Jiang, and H. B. Xu, J. Magn. Magn. Mater. 454, 97 (2018).

[22] L. Mañosa, S. Jarque-Farnos, E. Vives, and A. Planes, Appl. Phys. Lett. 103, 211904 (2013).

[23] J. Tušek, K. Engelbrecht, L. P. Mikkelsen, and N. Pryds, J. Appl. Phys. 117, 124901 (2015).

[24] H. A. Padilla, C. D. Smith, J. Lambros, A. J. Beaudoin, and I. M. Robertson, Metall. Mater. Trans. A 38, 2916 (2007).

[25] R. F. Hamilton, H. Sehitoglu, Y. Chumlyakov, and H. J. Maier, Acta Mater. 52, 3383 (2004).

[26] J. Cui, Y. S. Chu, O. O. Famodu, Y. Furuya, J. Hattrick-Simpers, R. D. James, A. Ludwig, S. Thienhaus, M. Wuttig, Z. Zhang, and I. Takeuchi, Nature Mater. 5, 286 (2006).

[27] X. Moya, S. Kar-Narayan, and N. D. Mathur, Nature Mater. 13, 439 (2014).

[28] X. Moya, E. Defay, V. Heine, and N. D. Mathur, Nat. Phys. 11, 202 (2015). 